\title{
Transfusion-related Acute Lung Injury: A Case Report
}

\author{
(D) Arzu Çalışkan Polat1, (1) Yeşim Yiğit2, (1) Esra Nagehan Akyol Önder33, (1) Ayşen Türedi Yıldırım², \\ ๑ Pelin Ertan33, ๑ Hüseyin Gülen2 \\ ${ }^{1}$ Celal Bayar University Faculty of Medicine, Department of Pediatrics, Manisa, Turkey \\ ${ }^{2}$ Celal Bayar University Faculty of Medicine, Department of Pediatric Hematology, Manisa, Turkey \\ ${ }^{3}$ Celal Bayar University Faculty of Medicine, Department of Pediatric Nephrology, Manisa, Turkey
}

\begin{abstract}
Transfusion-related acute lung injury (TRALI) is a non-cardiac pulmonary edema presenting with severe dyspnea, hypoxia and bilateral diffuse infiltrates on chest X-ray. It usually occurs within the first 6 hours following the administration of plasma and plasma rich blood products. Herein, we present a patient who developed TRALI shortly after plasmapheresis due to Hemolytic Uremic syndrome and exhibited a dramatic response with early systemic steroid treatment.
\end{abstract}

Keywords: Transfusion-related acute lung injury, hemolytic uremic syndrome, respiratory distress, plasmapheresis, systemic steroid treatment

\section{Introduction}

Transfusion-related acute lung injury (TRALI) is characterized by tachypnea, tachycardia, cyanosis and dyspnea and it usually occurs within the first 6 hours following the administration of plasma or plasma-rich blood products. It is diagnosed by acute lung injury starting during or within 6 hours of transfusion, lack of lung damage before transfusion and the exclusion of other causes that may lead to this condition (1). The incidence has been reported as 1 per 5,000 transfusions. Although it may develop due to all blood products, it has been reported that the transfusion of platelet suspensions which have been kept in a blood bank for a long time or blood products containing more than $50 \mathrm{~mL}$ of plasma increase the risk (2). Within transfusion reactions, it is the fourth most common one and also it is the third most common cause of mortality (3). TRALI is rarely diagnosed because it is often not considered among the preliminary diagnoses by clinicians.

\section{Case Report}

A 7-year-old girl with a history of recurrent diarrhea with bloody mucus every 15 minutes (20 times a day), and therefore being treated with metronidazole, was referred to us because of fatigue, skin color paleness and impaired renal function tests, although her diarrhea complaint regressed on the $3^{\text {rd }}$ day. When the patient arrived, there were no pathological findings except for a lethargic and pale appearance in physical examination. Blood tests revealed haemoglobin: $9.6 \mathrm{~g} / \mathrm{dL}$, Hematocrit: $28 \%$, mean corpuscular volume: $76.7 \mathrm{fL}$, white blood cells:

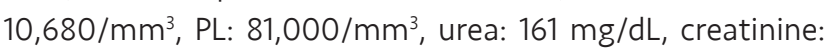
$4.9 \mathrm{mg} / \mathrm{dL}$, urea nitrogen: $75 \mathrm{mg} / \mathrm{dL}$, uric acid: $9.5 \mathrm{mg} / \mathrm{dL}$, total serum bilirubin: $1 \mathrm{mg} / \mathrm{dL}$, indirect bilirubin: $0.8 \mathrm{mg} /$

\section{Address for Correspondence}

Arzu Çalışkan Polat MD, Celal Bayar University Faculty of Medicine, Department of Pediatrics, Manisa, Turkey Phone: +90 5076055639 E-mail: arzucaliskan@outlook.com ORCID: orcid.org/0000-0002-0397-7074 Received: 01.09.2019 Accepted: 16.12.2019

${ }^{\circ}$ Copyright 2020 by Ege University Faculty of Medicine, Department of Pediatrics and Ege Children's Foundation The Journal of Pediatric Research, published by Galenos Publishing House. 
$\mathrm{dL}$, lactace dehydrogenase: 2,085 IU/L, albumin: $3.4 \mathrm{~g} / \mathrm{dL}$, total protein: $5.5 \mathrm{~g} / \mathrm{dL}$, alamine aminotransferase: $32 \mathrm{IU} / \mathrm{L}$, aspartate aminotransferase: $104 \mathrm{IU} / \mathrm{L}$. Serum electrolytes and venous blood gases were as follows; $\mathrm{Na}: 129 \mathrm{mEq} / \mathrm{L}$, $\mathrm{CL}: 99 \mathrm{mEq} / \mathrm{L}, \mathrm{K}: 4 \mathrm{mEq} / \mathrm{L}, \mathrm{Ca}: 8 \mathrm{mg} / \mathrm{dL}, \mathrm{pH}: 7.33$, and $\mathrm{HCO}_{3}$ : $19.5 \mathrm{mmol} / \mathrm{L}$, respectively. Direct Coombs test was negative. Urine analysis revealed the following: density: 1,024, $\mathrm{pH}: 5$, protein $(+++)$, blood $(+++)$. With the thrombocytopenia, hemolytic anemia and renal failure triad, and also previous complaints of bloody diarrhea, low hemoglobin and platelet counts, and peripheral blood smear findings (35\% spherocytes and schistocytes), Hemolytic Uremic syndrome (HUS) was diagnosed. The stool culture and shiga toxin producing $E$. coli were negative in the enteric bacterial panel. ADAMTS 13 activity level was detected to be normal at 94\%. Plasmapheresis and hemodialysis were planned with the preliminary diagnosis of atypical HUS. Blood was sent for mutation analyzes for atypical HUS. On the second day of hospitalization, the patient underwent hemodialysis for 30 minutes. Pre-hemodialysis biochemistry revealed creatinine: $5.22 \mathrm{mg} / \mathrm{dL}$, urea: $166 \mathrm{mg} / \mathrm{dL}$ and uric acid: $8.8 \mathrm{mg} / \mathrm{dL}$. After hemodialysis, they reduced as follows: creatinine: $4.27 \mathrm{mg} /$ $\mathrm{dL}$, urea: $110 \mathrm{mg} / \mathrm{dL}$, uric acid: $5.2 \mathrm{mg} / \mathrm{dL}$. One day later, plasmapheresis was performed with 4 units of fresh frozen plasma. There was no problem during plasmapheresis. However, one hour later, the patient developed respiratory distress, tachypnea, tachycardia and desaturation. The patient was provided with 6-10 L/min oxygen support. On physical examination, respiratory sounds were equal in both hemi thorax, but diminished bilaterally, rales-rhonchus was not detected, heart beats were rhythmic, and there were no murmur or other additional sounds and no edema-acid or hepatomegaly. Bilateral diffuse irregular reticulonodular infiltrates were detected on PA chest X-ray (Figure 1). Sudden respiratory failure after plasmapheresis was thought to be due to TRALI. Methylprednisolone (10 mg/kg/day) was

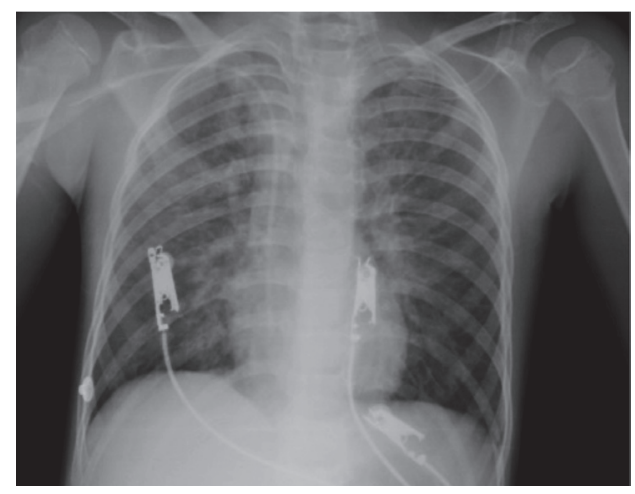

Figure 1. Chest X-Ray soon after plasmapheresis during respiratory distress administered intravenously for 2 days. During follow-up, respiratory distress regressed, oxygen necessity decreased and mechanical ventilation was not needed. Infiltrations regressed on PA chest X-ray (Figure 2). Monitoring with hemodialysis continued in the patient while respiratory distress regressed. Plasmapheresis was not repeated.

\section{Discussion}

Transfusion-related acute lung injury is a serious complication of blood products transfusion and is one of the most common causes of transfusion-related deaths. As clinicians do not consider this complication as a preliminary diagnosis, and therefore it is often not diagnosed, the actual frequency is not known but it is estimated to be 1 per 5,000 transfusions (4). Diagnosis is made by the development of acute lung injury during or within the first six hours of transfusion, the absence of acute lung injury prior to transfusion and the presence of risk factors that may lead to this condition (5).

TRALI is a non-cardiac pulmonary edema with severe dyspnea, hypoxia and bilateral diffuse infiltrations on chest $\mathrm{X}$-ray. The mechanism ofTRALI has not been clearly explained. According to the most accepted theory; the alloantibodies (anti-HLA class I and II, antineutrophil antibodies) from the donor activate the recipient's neutrophils, monocytes and tissue macrophages after the transfusion of plasmacontaining blood products. Activation of granulocytes initiates capillary damage, increases permeability and the inflammatory process. Fluid filling occurs in the alveolar space due to diffuse alveolar capillary damage. The detection of anti-HLA or anti-neutrophil antibodies is helpful in diagnosis (6).

Another theory suggests that biologically active lipids such as phosphatidylcholine released by the breakdown of cellular components in stored blood are also effective in TRALI

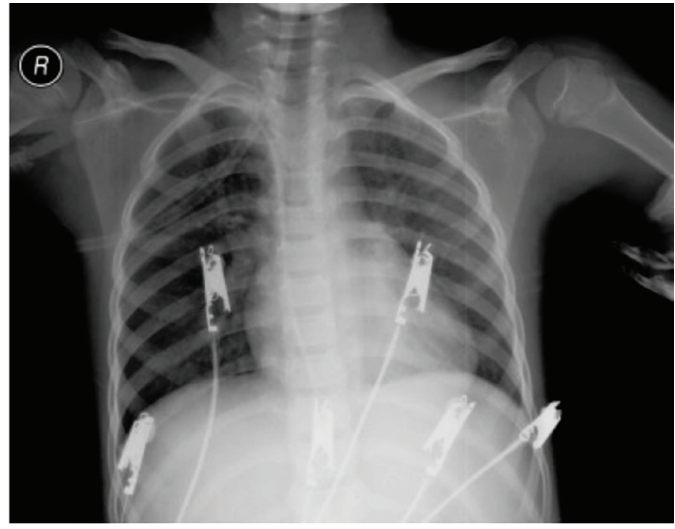

Figure 2. Chest X-ray taken 48 hours after plasmapheresis 
formation and that such lipids binding to "platelet activating factor" receptors cause damage to the pulmonary bed (7).

In this case, there was no respiratory distress prior to plasmapheresis and there was no other reason to explain acute lung injury that developed immediately after the procedure. Causes of cardiogenic pulmonary edema such as transfusion associated circulatory overload (TACO), congestive heart failure and myocardial infarction should be ruled out in patients with a possible diagnosis of TRALI (8). TACO with findings such as transient volume increase with transfusion, dyspnea, increased respiratory rate, hypoxemia and jugular venous fullness is the most confused situation with TRALI (9). Clinical signs of dyspnea, cyanosis, orthopnea, severe headache, hypertension and newly developing congestive heart failure during or immediately after transfusion are suggestive of the possibility of TACO. In the first evaluation of the patient, the presence of fullness in the jugular vein, gallop rhythm, and S3 should be checked. In our patient, plasmapheresis was performed with 4 units of TDP, and after 1 hour, next to observed respiratory distress, physical examination did not reveal hypertension, heart beats were rhythmic, there was no murmur or additional sounds, no signs of rapidly-developing coronary failure such as edema-acid-hepatomegaly, and no pathology in her electrocardiography (ECG). Oxygen supplementation was started with a mask and $10 \mathrm{mg} / \mathrm{kg} /$ day methylprednisolone was given intravenously for 2 days. Due to the rapid recovery of clinical findings within 24-48 hours, the absence of ischemia on ECG or dynamic troponin changes or other cardiac causes, our case was diagnosed as TRALI.

Since there is no specific treatment for TRALI, the main component of patient management is symptomatic and supportive treatment. As soon as the diagnosis of TRALI is considered, transfusion should be terminated immediately. Oxygen and hemodynamic support should be given. Some patients may require mechanical ventilation. The key to the management of these patients is the treatment of hypoxemia. Fluid therapy is also important for hypovolemia and hypotension and vasopressor agent support may be required. Therefore, diuretics should be avoided. The advantages or disadvantages of steroids have not been clarified (10). However, we think that in our case, we received a dramatic response to steroid treatment which was initiated as soon as symptoms began. After supportive therapy in TRALI, $80 \%$ of patients had symptom regression within the first 96 hours while 20\% required long-term supportive care. The mortality rate has been estimated to be $5-10 \%$. In our case, radiological and clinical improvement was achieved within 48 hours with respiratory-hemodynamic support and systemic steroid treatment.

\section{Conclusion}

Transfusion-related acute lung injury should be kept in mind in cases of acute respiratory distress during or immediately after transfusion of blood products containing plasma and if the transfusion is still in progress, it should be terminated immediately.

Ethics

Informed Consent: Informed consent from patient's family was received.

Peer-review: Internally peer-reviewed.

\section{Authorship Contributions}

Concept: Y.Y., E.N.A.Ö., A.T.Y., P.E., H.G., A.Ç.P., Data Collection or Processing: Y.Y., E.N.A.Ö., A.T.Y., P.E., H.G., A.Ç.P., Analysis or Interpretation: Y.Y., E.N.A.Ö., A.T.Y., P.E., H.G., A.Ç.P., Literature Search: Y.Y., E.N.A.Ö., A.T.Y., P.E., H.G., A.Ç.P., Writing: Y.Y., A.T.Y., H.G., A.Ç.P.

Conflict of Interest: No conflict of interest was declared by the authors.

Financial Disclosure: The authors declared that this study received no financial support.

\section{References}

1. Vlaar APJ, Juffermans NP. Transfusion-related acute lung injury: a clinical review. Lancet 2013; 382:984-94.

2. Goldman M, Webert KE, Arnold DM, et al. Proceedings of a consensus conference: Towards and understanding of TRALI. Transfus Med Rev 2005; 19:2-31.

3. Triulzi DJ. Transfusion-related acute lung injury: an update. Hematology Am Soc Hematol Educ Program 2006:497-501.

4. Popovsky MA, Davenport RD. Transfusionrelated acute lung injury: femme fatale? Transfusion 2001; 41:312-5.

5. Kleinman S, Caulfi eld T, Chan P, et al. Toward an understanding of transfusion-related acute lung injury: Statement of a consensus panel. Transfusion 2004; 44:1774-89.

6. Jawa RS, Anillo S, Kulaylat MN. Transfusion-related acute lung injury. I Intensive Care Med 2008; 23:109-21.

7. Silliman CC, Boshkov LK, Mehdizadehkashi Z et al. Transfusionrelated acute lung injury: epidemiology and a prospective analysis of etiologic factors. Blood 2003; 101:454-62.

8. Benson A, Moss M, Silliman C. Transfusion-related acute lung injury (TRALI): a clinical review with emphasis on the critically ill. BJH 2009; 174:431-43.

9. Murphy EL, Kwaan N, Looney MR, et al. Risk factors and outcomes in transfusion-associated circulatory overload. Am I Med. 2013; 126:357.e29-38.

10. Silliman CC, Fung YL, BradLey Ball ), Khan SY. Transfusion related acute lung injury: Cur-rent concepts and misconceptions. Blood Rev 2009; 23:245-55. 